\title{
Poland syndrome, a rare congenital disorder with no functional deficit: a case report
}

\author{
Jalaz Jain ${ }^{1 *}$, Priyanjal Jakhar², Ankit Mittal ${ }^{3}$, Ravi Sihag ${ }^{4}$, Kunal Bajaj ${ }^{5}$, Vibhuti Kumar ${ }^{6}$
}

\author{
${ }^{1}$ Department of Orthopaedics, Satyawadi Raja Harishchandra Hospital, Narela, New Delhi, India \\ ${ }^{2}$ Department of Orthopaedics, MAMC, Agroha, Hisar, Haryana, India \\ ${ }^{3}$ Department of Sports Medicine, ${ }^{4}$ Department of Orthopaedics, Pt. B. D. Sharma PGIMS, Rohtak, Haryana, India \\ ${ }^{5}$ Shivam Hospital, Rohini, New Delhi, India \\ ${ }^{6}$ Department of Anaesthesiology and Critical Care, Pt. B. D. Sharma PGIMS, Rohtak, Haryana, India
}

Received: 29 August 2020

Accepted: 14 September 2020

\author{
*Correspondence: \\ Dr. Jalaz Jain, \\ E-mail: jalazjain@gmail.com
}

Copyright: () the author(s), publisher and licensee Medip Academy. This is an open-access article distributed under the terms of the Creative Commons Attribution Non-Commercial License, which permits unrestricted non-commercial use, distribution, and reproduction in any medium, provided the original work is properly cited.

\begin{abstract}
Poland syndrome is a rare congenital syndrome. Most of the reported cases are sporadic, pattern of genetic inheritance is not known. It is an anomaly in which there is underdeveloped or absent pectoralis major and minor muscles leading to abnormal appearance of chest on the involved side. Most cases are unilateral with minimal functional abnormality in majority of the cases but with major cosmetic concerns for the patient. Surgery is rarely indicated but if required is done mainly for cosmetic purposes. We report a case of Poland syndrome in a young healthy individual as it started becoming evident.
\end{abstract}

Keywords: Poland syndrome, Chest wall, Pectoralis major

\section{INTRODUCTION}

Poland syndrome is named after Sir Alfred Poland. He gave a title of "deficiency of the pectoral muscles". ${ }^{1}$ It is a disorder in which there is absence or underdeveloped pectoral muscles resulting in abnormalities that can affect the chest, shoulder, arm and hand. Patients may present with alopecia of ipsilateral axilla and chest wall. ${ }^{2}$ Severely affected individuals have abnormalities in renal or cardiovascular system.

The exact mechanism of inheritance is unknown. Most cases of Poland syndrome are sporadic with an estimated incidence of $1: 36,000$ to $1: 50,000 .^{1}$ The severity of this disorder is variable and it may not become evident till puberty. It is a rare congenital disorder, hence we report a case of Poland syndrome in an 18 year old male who presented in outpatient department at our institute for routine medical examination for recruitment in Indian armed force.

\section{CASE REPORT}

We report a case of Poland syndrome in an 18 year old male who presented in our institute for regular routine medical examination as a part of recruitment in Indian armed forces. The chief complaint of the patient was abnormal appearance of the left side of his chest. On examination, the overlying skin was normal with a thinned subcutaneous fat layer. Nipple areolar complex was normal in appearance. There was an evident abnormality on the left side that appeared as visualisation of ribs directly under the skin on left side which was not the case on normal side (Figure 1a). There were no sinuses or scar marks or sign of any haemangiomas. There were no visible pulsations on the involved side. On palpation, the underlying ribs were readily palpable starting from second 
rib to ninth rib due to absence of overlying musculature and thinned out subcutaneous tissue. The temperature was comparable to normal side. The patient had full range of motion on the affected side- abduction, adduction, external rotation, internal rotation and forward elevation (Figure bd).

There was no weakness in adduction and internal rotation. On internal rotation and adduction, posterior wall of axilla became prominent due to absence of anterior axillary wall musculature (Figure 1e). Scapula became prominent on internal rotation of shoulder on affected side (Figure 1f). It was observed that patient was using his deltoid muscle to compensate for the loss of function of pectoral muscles. There was no functional deficit on the involved side in the patient and the patient was able to carry out all of his daily activities without any impairment. Magnetic resonance imaging of chest wall was done to rule out soft tissue abnormalities (Figure 2). It was observed in magnetic resonance imaging (MRI) of the chest that there was complete absence of pectoral muscles. Rib cage was normal as evident clinically and in chest radiograph (Figure 3).

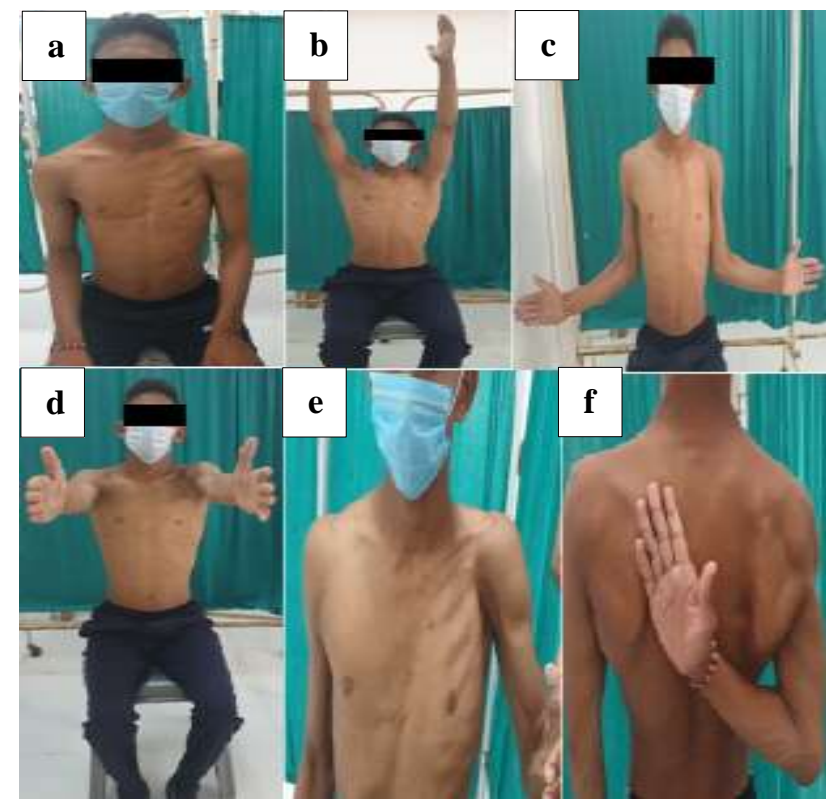

Figure 1: (a) Clinical image showing absence of pectoral muscles and visible ribs on left side of chest, (b) comparable abduction on both sides, (c) normal internal rotation of the affected side, (d) normal forward flexion, (e) prominent posterior wall of axilla on resisted internal rotation, and (f) scapular prominence on internal rotation.

Electrocardiography and echocardiogram were done to rule out any associated cardiovascular anomaly. Both of the investigations were normal. Patient had good exercise tolerance and metabolic equivalent score was more than four. Patient did not have any other history of chronic illness and insignificant childhood history.
No kyphosis or scoliosis was evident clinically. Spine radiographs in lateral and anteroposterior view were done to rule out any associated spinal deformities. All routine blood investigations were within normal limit. Patient was advised supportive management and referred to higher centre for genetic counselling and for further management.

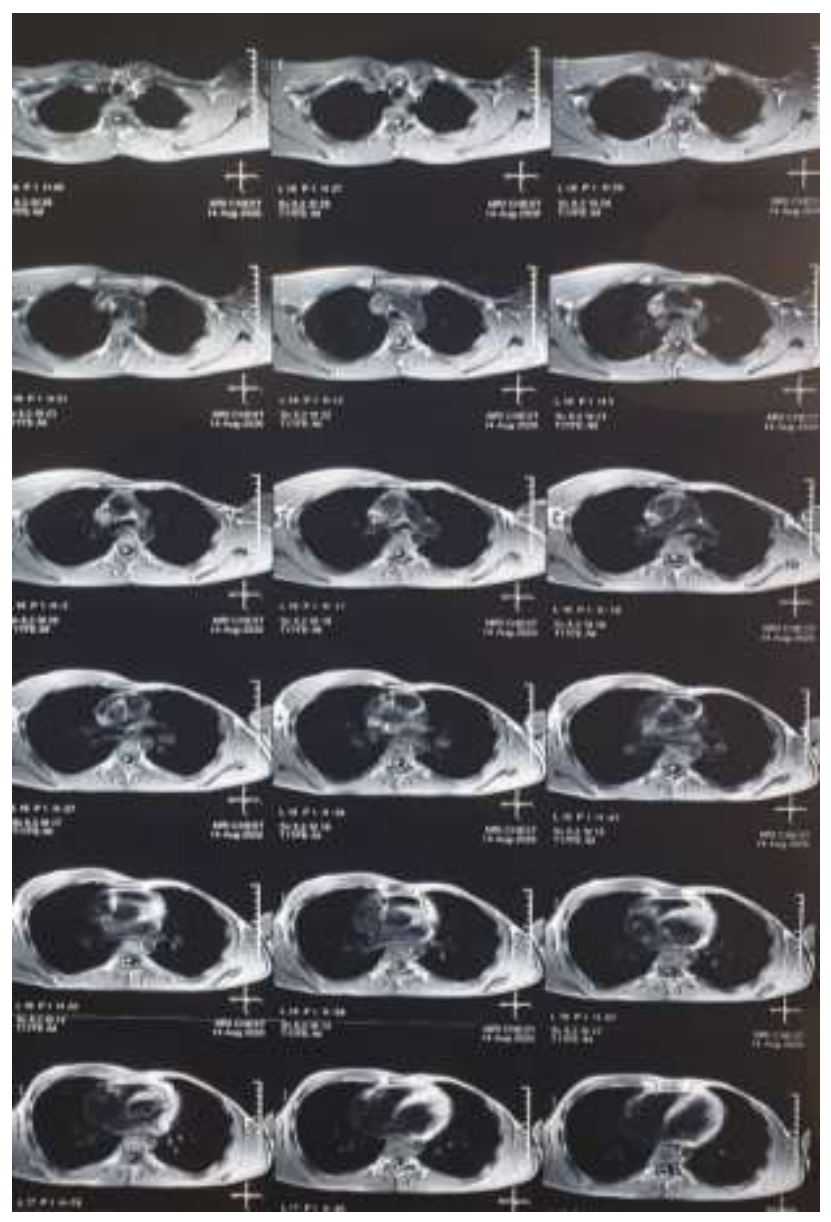

Figure 2: MRI chest showing complete absence of pectoralis muscles on left side.

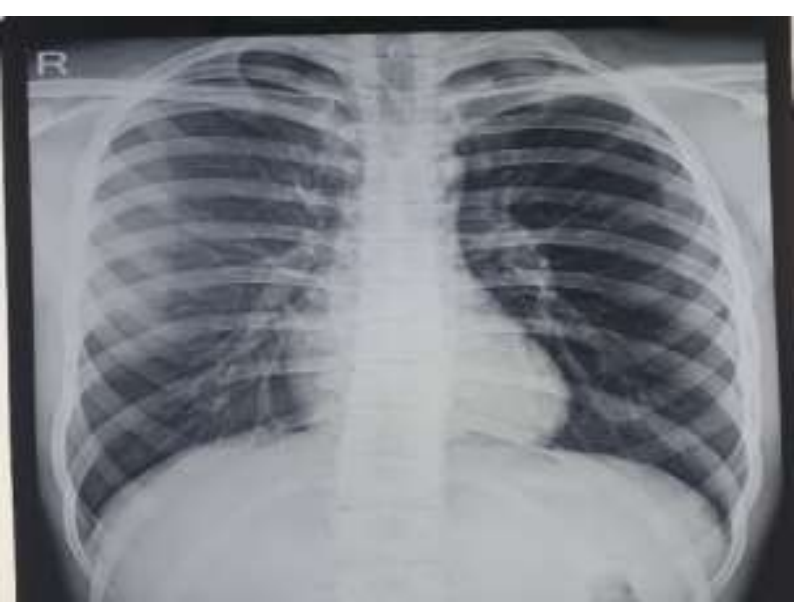

Figure 3: Normal chest radiograph anteroposterior view. 


\section{DISCUSSION}

People born with Poland anomaly have cosmetic disabilities which can be managed if correctly diagnosed. It becomes evident at birth due to asymmetry of chest. It is more common in males. ${ }^{1}$ It includes aplasia or hypoplasia of pectoralis major most commonly from the sternocostal origin associated with various abnormalities most commonly hypoplasia of pectoralis minor and ipsilateral 2 to 5 rib. ${ }^{3}$ Upper limb abnormality if present include small hand and or brachysyndactyly. ${ }^{4}$

There has been rare association with other congenital syndrome - Mobius syndrome and Pierre - Robin syndrome. ${ }^{5}$ Exact pathological cause is unknown. Most of the cases are sporadic. In our patient no such deformity was present in his siblings or in his ancestors. There was no associated cardiovascular abnormality or spinal deformity. There was no associated co morbidities.

There are few studies and case reports in literature on Poland syndrome. Deniz et al reported a 20 years old patient with Poland syndrome. ${ }^{6}$ The case had anomaly of shortness of right hand fingers and syndactyly between second and third fingers in addition to absence of right pectoralis muscle group. There was no another associated anomalities. Strength loss in abduction and adduction of right shoulder was detected. Baban et al reported a 3 and a half year old girl with bilateral Poland syndrome consisting of asymmetric pectoral muscle defects. ${ }^{7}$ There was complete agenesis of pectoral muscle on the left side and agenesis of the sternocostal head only on the right side, nipple hypoplasia, left rib defect, and right hand symbrachydactyly.

Patient in present study is student by occupation and wanted to pursue a career in armed forces. The patient is involved in regular exercises and lifts weights regularly for his fitness. The abnormality became more evident at puberty. Patient came for regular routine medical examination. As patient did not have any functional limitation, patient was referred to higher centre for genetic counselling and for further management.

Funding: No funding sources

Conflict of interest: None declared

Ethical approval: Not required

\section{REFERENCES}

1. Poland A. Deficiency of the pectoral muscle. Guys Hosp Rep. 1841;6:191.

2. Moir CR, Johnson CH. Poland's syndrome. Semin Pediatr Surg. 2008;17:161-6.

3. Fokin AA, Robicsek F. Poland's syndrome revisited. Ann Thorac Surg. 2002;74:2218-25.

4. Friedman T, Reed M, Elliott AM. The carpal bones in Poland syndrome. Skeletal Radiol. 2009;38:58591.

5. Pisteljić DT, Vranjesević D, Apostolski S, Pisteljić DD. Poland syndrome associated with 'morning glory' syndrome (coloboma of the optic disc). J Med Genet. 1986;23:364.

6. Deniz O, Tozkoparan E, Gümüş S. Poland syndrome (a case report). Tuberk Toraks. 2005;53:275-9.

7. Baban A, Torre M, Bianca S. Poland syndrome with bilateral features: case description with review of the literature. Am J Med Genet A. 2009;149:1597-602.

Cite this article as: Jain J, Jakhar P, Mittal A, Sihag R, Bajaj K, Kumar V. Poland syndrome, a rare congenital disorder with no functional deficit: a case report. Int J Res Orthop 2020;6:1306-8. 